\title{
Neurotoxic effects of MPTP on mouse cerebral cortex: Modulation of neuroinflammation as a neuroprotective strategy
}

\author{
Mariana Oliveira Mendes ${ }^{\mathrm{a}, 1}$, Alexandra Isabel Rosa ${ }^{\mathrm{a}, 1}$, Andreia Neves Carvalho ${ }^{\mathrm{a}}$, \\ Maria João Nunes $^{\mathrm{a}}$, Pedro Dionísio ${ }^{\mathrm{a}}$, Elsa Rodrigues, ${ }^{\mathrm{a}, \mathrm{d}}$, Daniela Costa ${ }^{\mathrm{a}}$, Sara Duarte-Silva ${ }^{\mathrm{b}, \mathrm{c}}$, \\ Patrícia Maciel $^{\mathrm{b}, \mathrm{c}}$, Cecília Maria Pereira Rodrigues ${ }^{\mathrm{a}, \mathrm{d}}$, Maria João Gama ${ }^{\mathrm{a}, \mathrm{d}}$, \\ Margarida Castro-Caldas ${ }^{\mathrm{a}, \mathrm{e}, *}$
}

${ }^{a}$ Research Institute for Medicines (iMed.ULisboa), Faculty of Pharmacy, Universidade de Lisboa, Av. Prof. Gama Pinto, 1649-003 Lisbon, Portugal

${ }^{\mathrm{b}}$ Life and Health Sciences Research Institute (ICVS), School of Medicine, University of Minho, 4710-057 Braga, Portugal

${ }^{c}$ ICVS/3B's PT Government Associate Laboratory, University of Minho, Guimarães, Braga, Portugal

${ }^{\mathrm{d}}$ Department of Biochemistry and Human Biology, Faculty of Pharmacy, Universidade de Lisboa, Av. Prof. Gama Pinto, 1649-003 Lisbon, Portugal

${ }^{\mathrm{e}}$ UCIBIO, Departamento Ciências da Vida, Faculdade de Ciências e Tecnologia, Universidade NOVA de Lisbon, Caparica, Portugal

\section{A R T I C L E I N F O}

\section{Keywords:}

Parkinson's disease

TUDCA

ANXA1

Neuroinflammation

Microglia

\begin{abstract}
A B S T R A C T
Parkinson's disease (PD) is a progressive neurological disorder, mainly characterized by the progressive loss of dopaminergic neurons in the Substantia nigra pars compacta (SNpc) and by the presence of intracellular inclusions, known as Lewy bodies. Despite SNpc being considered the primary affected region in PD, the neuropathological features are confined solely to the nigro-striatal axis. With disease progression other brain regions are also affected, namely the cerebral cortex, although the spreading of the neurologic damage to this region is still not completely unraveled.

Tauroursodeoxycholic acid (TUDCA) is an endogenous bile acid that has been shown to have antioxidant properties and to exhibit a neuroprotective effect in 1-methyl-4-phenyl-1,2,3,6-tetrahydropyridine (MPTP) mice model of PD. Moreover, TUDCA anti-inflammatory properties have been reported in glial cells, making it a prominent therapeutic agent in PD.

Here, we used C57BL/6 mice injected with MPTP in a sub-acute paradigm aiming to investigate if the neurotoxic effects of MPTP could be extended to the cerebral cortex. In parallel, we evaluated the anti-oxidant, neuroprotective and anti-inflammatory effects of TUDCA. The anti-inflammatory mechanisms elicited by TUDCA were further dissected in microglia cells.

Our results show that MPTP leads to a decrease of ATP and activated AMP-activated protein kinase levels in mice cortex, and to a transient increase in the expression of antioxidant downstream targets of nuclear factor erythroid 2 related factor 2 (Nrf-2), and parkin. Notably, MPTP increases pro-inflammatory markers, while down-regulating the expression of the anti-inflammatory protein Annexin-A1 (ANXA1). Importantly, we show that TUDCA treatment prevents the deleterious effects of MPTP, sustains increased levels of antioxidant enzymes and parkin, and most of all negatively modulates neuroinflammation and up-regulates ANXA1 expression. Additionally, results from cellular models using microglia corroborate TUDCA modulation of ANXA1 synthesis, linking inhibition of neuroinflammation and neuroprotection by TUDCA.
\end{abstract}

\section{Introduction}

Parkinson's disease (PD) is the second most common neurodegenerative disorder, affecting about $3 \%$ of the population over 65 years of age and $0.3 \%$ of the overall worldwide population (Pringsheim et al.,
2014). PD is mainly a motor disorder, pathologically characterized by the loss of dopaminergic neurons in the Substantia nigra pars compacta $(\mathrm{SNpc})$, severe striatal dopamine depletion, and by the presence of intracellular inclusions containing aggregates of $\alpha$-synuclein known as Lewy bodies (Goedert et al., 2013; Przedborski, 2005).

\footnotetext{
* Corresponding author at: Research Institute for Medicines (iMed.ULisboa), Faculty of Pharmacy, Universidade de Lisboa, Av. Prof. Gama Pinto, 1649-003 Lisboa, Portugal.

E-mail address: mcastrocaldas@ff.ulisboa.pt (M. Castro-Caldas).

${ }^{1}$ Mariana Oliveira Mendes and Alexandra Isabel Rosa are joint first authors.
} 
Despite extensive research, the mechanisms underlying neuropathology of PD are still largely unknown. However, it is believed that multiple genetic and environmental factors play critical roles in the development of the disease. The study of familial forms of PD, gave a decisive contribution to the unraveling of the molecular pathways involved in dopaminergic cell loss, including $\alpha$-synuclein proteostasis, mitochondrial dysfunction, oxidative stress, and neuroinflammation (Blesa et al., 2015; Klein and Westenberger, 2012; Moon and Paek, 2015; Niranjan, 2014).

The involvement of mitochondrial dysfunction in PD was reinforced by the discovery that exposure to 1-methyl-4-phenyl-1,2,3,6-tetrahydropyridine (MPTP) a selective inhibitor of mitochondrial Complex I, leads to parkinsonism and degeneration of dopaminergic neurons in humans and in animal models of the disease (Fabre et al., 1999; Nicklas et al., 1985; Rosa et al., 2018). In accordance, post mortem studies in PD patients brains showed reduced Complex I activity and increased reactive oxygen species (ROS) production (Hwang, 2013). Several mutations in familial PD also reaffirm the implication of mitochondrial dysfunction in this disease. For example, half of familial PD cases, and about $20 \%$ of the cases with early onset, are associated with loss-offunction mutations in the PARK2 gene. This gene encodes for parkin, a component of an E3 ubiquitin ligase complex, with an important role in the modulation of different aspects of mitochondrial turnover and homeostasis (Martin et al., 2011; Klein and Westenberger, 2012; Thomas and Flint, 2007).

Neuroinflammation involvement in PD pathogenesis was proven by the presence of reactive microglia and astrocytes, as well as increased levels of cytokines and pro-inflammatory enzymes in PD patients brains (McGeer et al., 1988; Mogi et al., 1994; Blum-Degen et al., 1995; Muller et al., 1998; Miklossy et al., 2006; Hirsch and Hunot, 2009; Liddelow et al., 2017). Moreover, serum and cerebrospinal fluid from PD patients, showed increased levels of cytokines (Hirsch and Hunot, 2009), further supporting an inflammatory contribution to the phenotype of this disease. We and others have also described the presence of reactive glia and pro-inflammatory mediators in both mouse and monkey experimental PD models (Hurley et al., 2003; Liberatore et al., 1999; Rosa et al., 2018).

PD studies are generally focused in the loss of dopaminergic neurons in the nigro-striatal axis. However, other brain areas are also affected in this disorder. In early stages, neuron loss is thought to be confined to the ventrolateral substantia nigra leaving other midbrain areas exempt, though it becomes more widespread by the end-stage of the disease (Dauer and Przedborski, 2003; Poewe et al., 2017). However, the spread of the neurologic insult to other brain regions besides the nigrostriatal axis is still not completely characterized. Therefore, understanding the broader damage, as well as the effects of therapeutic strategies in other areas is essential to fully characterize the pathogenic mechanisms involved in PD.

The discovery of new pharmacological approaches capable to delay, stop or revert neurodegeneration and PD progression are an urgent matter. Tauroursodeoxycholic acid (TUDCA) is an endogenous hydrophilic bile acid formed in the liver by conjugation of ursodeoxycholic acid (UDCA) with taurine. TUDCA is quickly bioavailable via oral, subcutaneous, or intravenous administration, has no associated toxicity and crosses the blood brain barrier (Ackerman and Gerhard, 2016; Rodrigues et al., 2002). Importantly, we showed that TUDCA treatment effectively prevented/ameliorated motor impairments of mice injected with MPTP (Rosa et al., 2018). Moreover, TUDCA also displayed an anti-inflammatory role by decreasing glia activation and pro-inflammatory markers expression while preventing the MPTP-induced decrease of annexin-A1 (ANXA1), prevented dopaminergic cell loss, mitochondrial dysfunction, ROS production and increased the expression of antioxidant enzymes in MPTP-injected mice midbrain and striatum (Castro-Caldas et al., 2012; Moreira et al., 2017; Rosa et al., 2017; Rosa et al., 2018).

Herein we used a sub-acute paradigm of MPTP administration in mice aiming to investigate if the neurotoxic effects, as well as the neuroprotection afforded by TUDCA, are extended to the cerebral cortex. In addition, we addressed the ability of TUDCA to modulate ANXA1 expression, in the cerebral cortex and in microglia cells, as part of its anti-inflammatory mechanisms.

\section{Material and methods}

\subsection{Ethics statement}

All procedures were conducted in accordance with European regulations (European Union Directive 2010/63/EU). Animal facilities and the people directly involved in animal experiments were certified by the Portuguese regulatory entity — Direção Geral de Alimentação e Veterinária.

\subsection{Animal treatments}

Twelve-week-old male C57BL/6J mice were purchased from Charles River and housed in groups of 4 or 5 animals in filter-topped polysulfone cages in a conventional animal facility. All animals were maintained under standard laboratory conditions: an artificial $12 \mathrm{~h}$ light/dark cycle, with an ambient temperature of $21 \pm 1^{\circ} \mathrm{C}$ and a relative humidity of $50-60 \%$; the mice were given a standard diet and water ad libitum. Health monitoring was performed according to FELASA guidelines (Nicklas et al., 2002; Guillen, 2012). Humane endpoints for experiments were defined (20\% reduction of the body weight, inability to reach food and water, presence of wounds in the body, dehydration), but not needed in practice, as animals did not reach these endpoints in the study period.

Mice were intraperitoneally (i.p.) injected once with MPTP (Sigma Aldrich), with a dose of $40 \mathrm{mg} / \mathrm{kg}$ body weight. This represents an acute MPTP paradigm used as an experimental model of drug-induced parkinsonism. This was previously shown by us and others to accurately recapitulate time-dependent analysis of nigro-striatal neuroadaptive changes (Jackson-Lewis et al., 1995; Saporito et al., 2000; JacksonLewis and Przedborski, 2007; Castro-Caldas et al., 2009; Rosa et al., 2018). TUDCA (Sigma Aldrich) was administered daily i.p. at a dose of $50 \mathrm{mg} / \mathrm{kg}$ body weight, for 3 consecutive days, as previously described (Keene et al., 2001; Castro-Caldas et al., 2012).

Mice designated for the short-term treatment were divided in 4 groups (6 mice per group): i) mice that received saline (Control, C); ii) mice injected with MPTP and euthanized $3 \mathrm{~h}$ after MPTP administration (MPTP); iii) mice that received daily injection of TUDCA during 3 consecutive days, followed by i.p. administration of MPTP on day $3,6 \mathrm{~h}$ after the last TUDCA injection, and that were euthanized $3 \mathrm{~h}$ after MPTP administration (TUDCA + MPTP); iv) mice injected with MPTP, followed by 3 daily injections of TUDCA starting $3 \mathrm{~h}$ after the neurotoxin administration and were euthanized $6 \mathrm{~h}$ after the last TUDCA administration (MPTP + TUDCA).

Mice evaluated in the long-term treatment were also divided in 4 groups (6 mice per group): i) mice that received saline (Control, C); ii) mice injected with MPTP and euthanized 52 days after MPTP administration (MPTP); iii) mice that received daily injection of TUDCA during 3 consecutive days, followed by i.p. administration of MPTP on day $3,6 \mathrm{~h}$ after the last TUDCA injection, and that were euthanized 52 days after MPTP administration (TUDCA + MPTP); iv) mice injected with MPTP, followed by 3 daily injections of TUDCA starting $3 \mathrm{~h}$ after the neurotoxin administration and were euthanized 52 days after MPTP injection (MPTP + TUDCA).

Treatments were carried out in at least 4 independent experiments $(n=4)$ for short- and long-term evaluation experiences.

After treatments, mice were decapitated, under anesthesia with sodium pentobarbital $(50 \mathrm{mg} / \mathrm{kg}$, i.p.), and the cerebral cortex was isolated at the level of the nigro-striatal axis (Bregma -3.20 to 1.00). Cortex region samples were stored at $-80^{\circ} \mathrm{C}$ until further use. 


\subsection{Culture conditions and cells treatments}

In vitro studies were carried out using the immortalized murine microgial cell line BV2 (ATCC). Cells were maintained in RPMI 1640 medium supplemented with $10 \%$ FBS and $1 \%$ of Penicillin/streptomycin, at $37^{\circ} \mathrm{C}$ in a humidified atmosphere of $5 \% \mathrm{CO}_{2}$, in HERAcell 150 incubators (Thermo Scientific, Waltham, MA, USA). Cells $\left(1 \times 10^{6}\right)$ were plated in $60 \mathrm{~mm}$ culture dishes and left to stabilize for $24 \mathrm{~h}$ prior to treatment with $100 \mu \mathrm{M}$ of TUDCA for different time points $(3,6$ or $24 \mathrm{~h}$ ). In another set of experiments BV2 cells were pre-treated with $5 \mu \mathrm{M}$ of $\mathrm{H} 89$ (an inhibitor of protein kinase A, PKA) for $1 \mathrm{~h}$, and then with $100 \mu \mathrm{M}$ of TUDCA for 3 or $6 \mathrm{~h}$ more. Controls were always included, and consisted of treating the cells with vehicle. Treatments were carried out in at least three independent experiments.

\subsection{Preparation of protein extracts}

Mice cortex protein extracts were prepared as previously described (Rosa et al., 2018).

After treatments, BV2 cells were washed with ice-cold PBS supplemented with or without $1 \mathrm{mM}$ of EDTA in order to seize extracellular ANXA1 (Castro-Caldas et al., 2003), or for preparation of total cell extracts, respectively. Subsequently, the preparation of intracellular or total cell extracts cells followed a previously described protocol (Rosa et al., 2017).

Total protein concentration was determined using Bio-Rad's Protein Assay Reagent, according to the manufacturer's instructions.

\subsection{Measurement of ATP levels}

ATP levels were measured in samples of mice cortex tissue extracts with the ATP-Glo ${ }^{\mathrm{TM}}$ Bioluminometric Cell Viability Assay (Biotium, Hayward, CA, USA), according to the manufacturer's instructions. The Luciferase activity in each sample was then measured in a luminometer (Berthold Systems). Negative controls were always included, and results were normalized to the total protein concentration in each well, as previously described (Rosa et al., 2017).

\subsection{Western blot analysis}

Protein extracts, from mice cortex or BV2 cells were separated in SDS-PAGE and electrotransfered to activated Immobilon P membranes (Millipore, Bedford, MA, USA).

The membranes were then blocked with $5 \%$ non-fat dry milk in TBS$\mathrm{T} 0.1 \%$, and then incubated with the specific primary antibodies depicted in Table 1 , overnight at $4{ }^{\circ} \mathrm{C}$ with agitation. Next, membranes were incubated with horseradish peroxidase-conjugated anti-mouse or anti-rabbit (Bio-Rad), or anti-Armenian hamster (Santa Cruz) secondary antibodies, for $1 \mathrm{~h}$ at r.t. The immunocomplexes were visualized by chemiluminescent detection with SuperSignal ${ }^{\circledR}$ West Femto Maximum Sensivity Substrate in a ChemiDoc ${ }^{\mathrm{TM}}$ MP imaging system from Bio-Rad Laboratories. After the evaluation of the different proteins, membranes were stripped and then incubated with anti- $\beta$-actin primary antibody as a loading control.

The relative intensities of protein bands were analyzed with Image Lab TM analysis software (Bio-Rad Laboratories, Hercules, CA, USA) and results were normalized to the percentage of control.

\subsection{Immunohistochemistry}

Cryostat coronal sections at the level of midstriatum and SNpc were prepared as previously described (Rosa et al., 2018). Incubation with primary antibodies anti-Ionized calcium binding adaptor molecule 1 (Iba-1) or anti-Glial fibrillary acidic protein (GFAP) (Table 1) was performed overnight at $4{ }^{\circ} \mathrm{C}$, followed by incubation with the antirabbit Alexa Fluor ${ }^{\circledR} 488$ secondary antibody (ThermoFisher Scientific), $1 \mathrm{~h}$ at r.t. Sections were observed under an Axioskop microscope (Carl Zeiss) with an attached Leica DFC490 camera, and photographed using Image Manager 50 software (Leica Microsystems, Inc.). Green (for both GFAP and Iba-1) and blue (for nuclei) fluorescence and UV images of, at least, 15 random microscopic fields belonging to mice cerebral cortex region, were acquired per slice, under $400 \times$ magnification. The results were expressed as the percentage of area occupied by GFAP or Iba-1 positive cells, measured by using the ImageJ software analysis (National Institutes of Health, USA).

Control experiments for nonspecific binding were performed in parallel by omission of the primary antibody.

\subsection{Statistical analysis}

All results are expressed as mean \pm SEM values. Data were analyzed by Student's $t$-test ( 2 groups), or one way ANOVA ( $>2$ groups) and differences between groups were determined by post hoc Bonferroni's test, whereas comparison of treatments to the control was performed by post hoc Dunnett's test, using GraphPad Prism 6.0 (San Diego, CA, USA). Means were considered statistically significant at a $p$ value $<0.05$
A

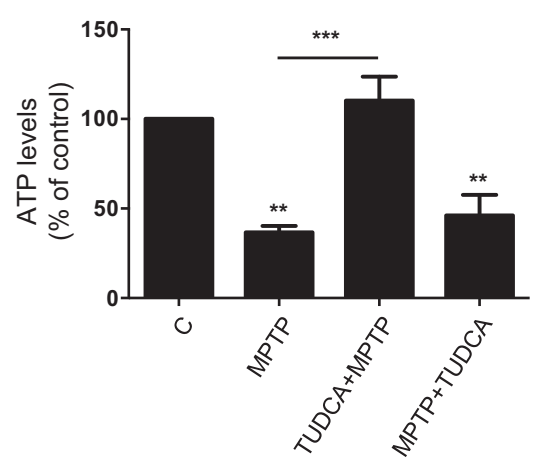

B

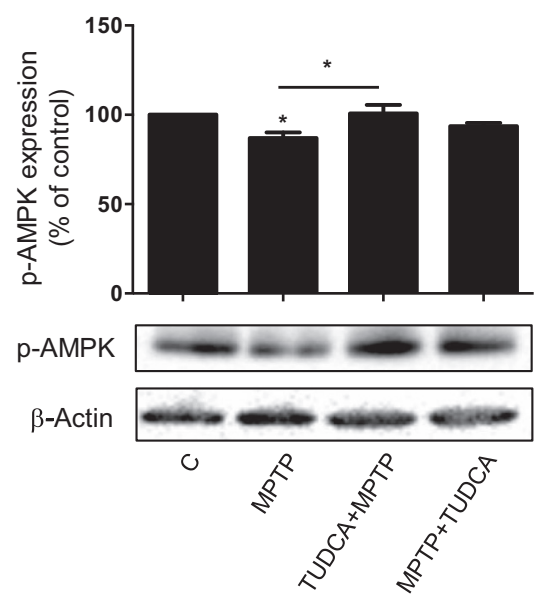

Fig. 1. ATP and p-AMPK levels in the cortex of MPTP-treated mice. Animals were treated with vehicle, MPTP, TUDCA prior to MPTP (TUDCA + MPTP) or TUDCA after MPTP (MPTP + TUDCA), and were euthanized 52 days post-MPTP administration. (A) ATP levels were quantified in cortex extracts. (B) Levels of p-AMPK were determined in the cortex by Western blot analysis. $\beta$-Actin was used as loading control. Relative band intensity was quantified using the Image Lab ${ }^{\mathrm{TM}}$ software and expressed as a percentage of control. Data represent mean values \pm SEM of at least three independent experiments, indicated as percentage of control. ${ }^{*} p<0.05,{ }^{* *} p<0.01$ and $* * * p<0.001$ using one-way ANOVA with Bonferroni's post hoc test. 


\section{Results}

\subsection{Effect of TUDCA on ATP levels and AMPK phosphorylation in the cortex of MPTP-treated mice}

To characterize the effects triggered by MPTP in the cortex, we determined ATP levels in mouse cortical extracts (Fig. 1A). As expected, upon a short-term treatment with MPTP we could not observe any changes in ATP levels in any condition studied (data not shown). However, at 52 days after MPTP injection there was a significant decrease of ATP levels as compared to controls. Importantly, pre-treatment with TUDCA prevented the decrease of ATP levels found in MPTPexposed animals. Mice that were post-treated with TUDCA showed ATP levels similar to those found in MPTP-injected animals.

To further evaluate the putative protective role of TUDCA on the ATP deficit induced by MPTP, we evaluated the phosphorylation levels of the energy sensor AMP-activated protein kinase (AMPK) (Fig. 1B). No changes in p-AMPK levels could be observed $3 \mathrm{~h}$ after MPTP administration (data not shown). Interestingly, results show that in the cortex of MPTP-injected animals for 52 days the levels of activated AMPK (p-AMPK) were significantly lower than in control mice. p-AMPK levels were significantly increased in animals treated with TUDCA before MPTP compared to MPTP alone. However, post-treatment with TUDCA did not change the phosphorylation status of AMPK.

\subsection{Effect of TUDCA on antioxidant enzymes in the cortex of MPTP- treated mice}

To evaluate whether treatment with MPTP/TUDCA was able to affect the expression of enzymes involved in the antioxidant response in the cortex of MPTP mice, we determined the levels of Glutathione peroxidase (GPx) and Heme oxygenase-1 (HO-1) in cortical extracts (Fig. 2). In animals euthanized $3 \mathrm{~h}$ post-MPTP administration (shortterm treatment), the levels of GPx were significantly increased in MPTP and in TUDCA + MPTP treated mice (Fig. 2A). However, when animals were euthanized 52 days after MPTP administration (long-term treatment) the increase in GPx expression was only maintained in animals pre-treated with TUDCA (Fig. 2B). HO-1 expression followed a trend similar to GPx after MPTP administration. However, pre-treatment with TUDCA significantly up-regulated HO-1 levels in short-term treatment, and preserved HO-1 concentration significantly higher than that observed in MPTP animals, in long-term experiments (Fig. 2A, B). Interestingly, although post-treatment with TUDCA at the longer time-point significantly decreases GPx expression, HO-1 expression remained high in these animals, suggesting different ways of activation for both enzymes.

\subsection{Effect of TUDCA on parkin levels in the cortex of MPTP mice}

We have previously shown that pre-treatment with TUDCA promotes mitophagy in MPTP-insulted mice via the E3 protein-ubiquitin ligase parkin (Rosa et al., 2017). Here we evaluated the effect of TUDCA, either before or after MPTP administration, on parkin expression in the cortex of animals euthanized $3 \mathrm{~h}$ or 52 days after MPTP injection. Results presented in Fig. 3A show that at shorter time-points, MPTP induced a significant increase in parkin levels. Post-treatment with TUDCA induced an increase of parkin similar to MPTP only, whereas pre-treatment with TUDCA exacerbated this increase. Up-regulation of parkin levels in response to MPTP seems to be a transient event, since at day 52 after MPTP administration only in animals that were pre-treated with TUDCA parkin protein levels were maintained significantly higher.

\subsection{Role of TUDCA on MPTP-induced neuroinflammation in the cortex}

Glial activation in the cortex was evaluated in animals euthanized 52 days after MPTP injection by determination of GFAP (Fig. 4A) or Iba1 (Fig. 4B) expression, markers for astrogliosis and microgliosis, respectively. In the cortex of MPTP-injected mice, in the presence or absence of TUDCA, GFAP and Iba-1 expression were significantly increased. Importantly, astrocyte activation was partially prevented in animals pre- or post-treated with TUDCA (Fig. 4A), whereas pre-treatment (but not post-treatment) with TUDCA partially prevented MPTPinduced Iba-1 expression (Fig. 4B).

To better evaluate the role of MPTP and/or TUDCA on neuroinflammation, levels of the pro-inflammatory cytokine interleukin-1 $\beta$ (IL1ß) (Fig. 4C) and of the anti-inflammatory protein ANXA1 (Fig. 4D) were determined by Western blot in cortex samples from these animals. Results show that IL-1 $\beta$ expression levels were significantly increased in MPTP animals, and that this increase was prevented by TUDCA
A
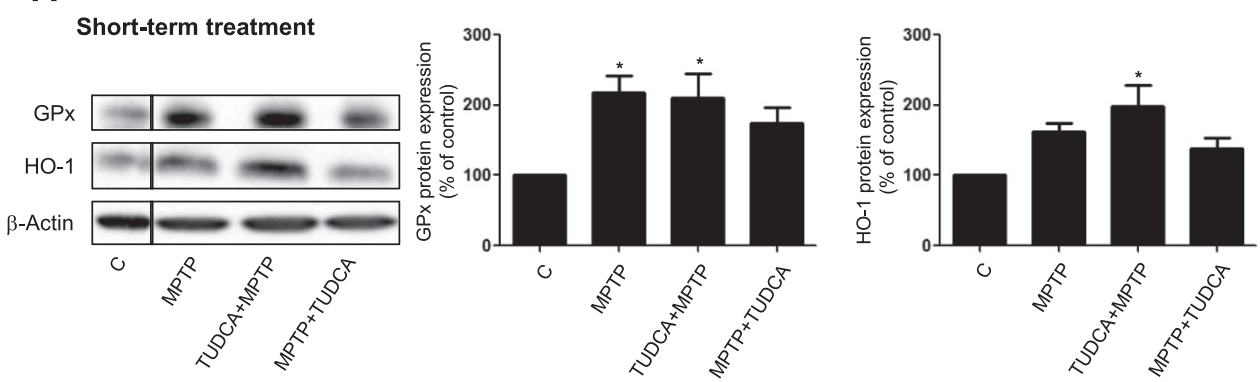

B
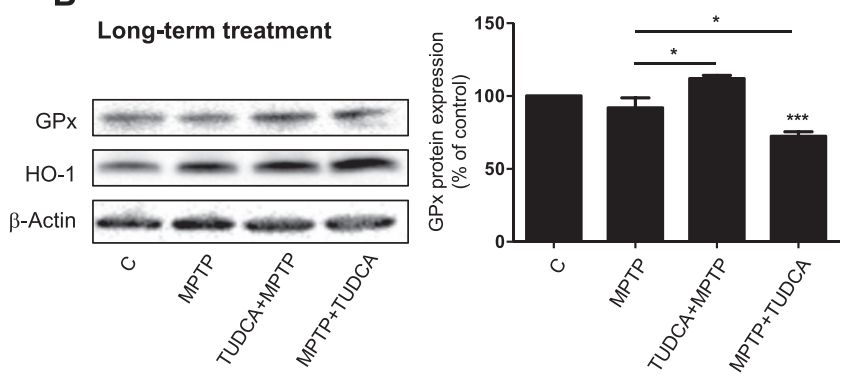

Fig. 2. TUDCA increases levels of antioxidant enzymes in the cortex of MPTPtreated mice. Mice were treated with vehicle, MPTP, TUDCA prior to MPTP (TUDCA + MPTP) or TUDCA after MPTP (MPTP + TUDCA), and were euthanized $3 \mathrm{~h}$ (Short-term treatment) (A) or 52 days (Longterm treatment) (B) post-MPTP administration. Antioxidant enzymes GPx and HO-1 were evaluated by Western blot analysis in the cortex extracts. $\beta$-Actin was used as loading control. Representative immunoblots are presented for each protein. Relative band intensity was quantified using the Image $\mathrm{Lab}^{\mathrm{TM}}$ software and expressed as a percentage of control. Data shown are mean values \pm SEM of at least three independent experiments. ${ }^{*} p<0.05$, and ${ }^{* * *} p<0.001$ using one-way ANOVA with Bonferroni's post hoc test. 
A

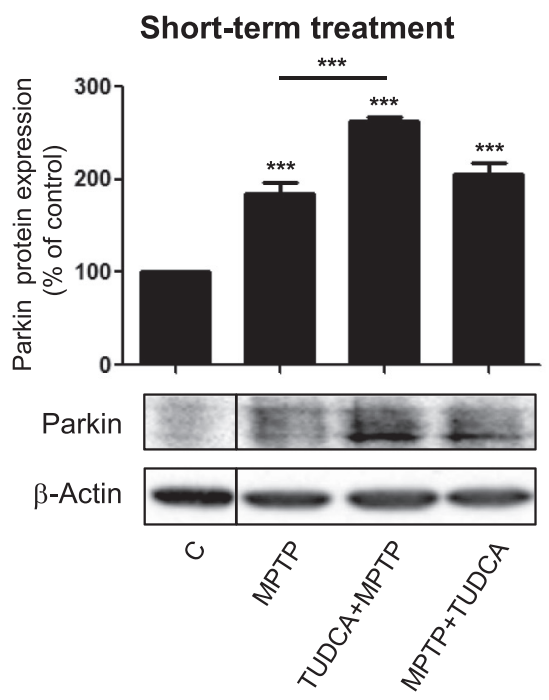

B

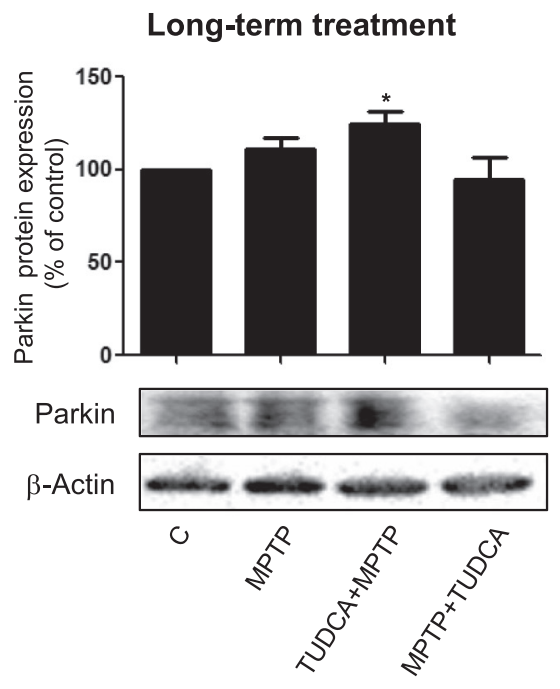

Fig. 3. TUDCA modulates the expression of parkin in the cortex of mice treated with MPTP. Mice were treated with vehicle, MPTP, TUDCA prior to MPTP (TUDCA + MPTP) or TUDCA after MPTP (MPTP + TUDCA), and were euthanized $3 \mathrm{~h}$ (Short-term treatment) (A) or 52 days (Longterm treatment) (B) post-MPTP administration. Representative immunoblots and respective quantification of parkin in cortex extracts are shown. $\beta$-Actin was used as loading control. Relative band intensity was quantified using the Image Lab $^{\mathrm{TM}}$ software and expressed as a percentage of control. Data represent mean values \pm SEM of at least three independent experiments, indicated as percentage of control. * $p<0.05$, $* * p<0.01$ and $* * * p<0.001$ using oneway ANOVA with Bonferroni's post hoc test. administration either before or after administration of the neurotoxin. On the other hand, ANXA1 expression levels were significantly increased in animals treated with TUDCA prior to or after MPTP injection.

Together, these results show that MPTP induces a pro-inflammatory response in the cortex that is efficiently ameliorated when animals are treated with TUDCA either before or after the neurotoxin administration.

\subsection{TUDCA modulation of ANXA1 expression in BV2 microglia cell line}

Our results show that neuroinflammation is a striking outcome of MPTP effects on mice cerebral cortex. We have also found that TUDCA treatment is, at least partially protective, by significantly decreasing neuroinflammation. ANXA1 seems to be an interesting target of TUDCA anti-inflammatory action. In order to further explore the mechanism by which this bile acid modulates ANXA1 expression we have used an in vitro glial cell model. Since in the brain ANXA1 is found to be almost exclusively expressed in glial cells and mainly in microglia (Solito et al., 2008), here we used the BV2 microglia cell line. In addition to evaluating intracellular levels of ANXA1, we also determined membranebound extracellular levels, considering the extracellular role of this protein in inflammatory processes. The intra- and extracellular levels of ANXA1 in response to treatment with TUDCA were evaluated at different time-points. Intracellular ANXA1 protein levels (Fig. 5A) were significantly higher $6 \mathrm{~h}$ after TUDCA administration. Interestingly, the extracellular levels of ANXA1 were also significantly increased after $6 \mathrm{~h}$ exposure to TUDCA (Fig. 5B). Together these results indicate that TUDCA induces the secretion of ANXA1 and replenishes the intracellular content of the protein, strongly suggesting de novo synthesis of ANXA1. Since the peak of intra- and extracellular protein was found at $6 \mathrm{~h}$ post-TUDCA addition, this time-point was used in the subsequent experiments aiming to determine the mechanisms underlying the modulation of ANXA1 expression by TUDCA.

\subsection{Unraveling the mechanism underlying the modulation of ANXA1 by TUDCA}

The promotor region of ANXA1 gene contains a cAMP responsive element (CRE) that requires an activated CRE binding protein (CREB) to induce ANXA1 mRNA synthesis (Antonicelli et al., 2001). CREB activation occurs upon its phosphorylation, a process regulated by several signaling pathways. cAMP is thought to play a critical role in this process, since production of this secondary messenger activates PKA, which is responsible for CREB phosphorylation (Hagiwara et al., 1993). Taking this into account, and together with the fact that TUDCA increases cAMP levels in primary cultures of microglia cells (YanguasCasas et al., 2017), we evaluated whether the activation of PKA pathway by TUDCA is involved in ANXA1 increased synthesis, using a specific pharmacological inhibitor of this kinase (H89). TUDCA treatment increased the levels of activated PKA (p-PKA), which were significantly prevented by pre-treatment with $\mathrm{H} 89$ (Fig. 5C). Both PKA and protein kinase B (AKT) have been implicated in CREB activation (Peltier et al., 2007). We already demonstrated that TUDCA modulates AKT activation in several models of disease, which is important for its neuroprotective effect (Solá et al., 2003; Castro et al., 2004; CastroCaldas et al., 2012). Here, we confirmed that TUDCA increased p-AKT levels in microglia, particularly at $6 \mathrm{~h}$ post-TUDCA (Fig. 5D), further corroborating the ability of TUDCA to modulate this pathway in different cellular contexts. Accordingly to the above, TUDCA increased the levels of p-CREB in a time-dependent manner, being statistically different at $6 \mathrm{~h}$ of treatment, indicating activation of this transcription factor. As expected, pre-treatment with $\mathrm{H} 89$ prevented the TUDCA-induced increase in p-CREB levels (Fig. 5E). Finally, the expression levels of total ANXA1 were significantly decreased when cells were treated with $\mathrm{H} 89$ prior to TUDCA (Fig. 5F). These results strongly suggest that TUDCA up-regulates ANXA1 expression in microglia through the activation of the PKA-CREB pathway.

In conclusion, in vitro results show that TUDCA modulates synthesis of ANXA1 in microglia cells, reinforcing the results obtained in mice cortex, and suggest that the anti-inflammatory effect of TUDCA may be, at least partially, mediated by ANXA1.

\section{Discussion}

The most vulnerable region in PD is considered to be the nigrostriatal axis and only a very limited number of studies have described PD damage progression in regions such as cerebral cortex (Cassarino et al., 1997; Fahn, 2003; Garcia-Esparcia et al., 2018; Becker et al., 2018), which is essential to address the development of new and more efficient therapeutic strategies.

Previous works showed that in early stages of the disease, neuron loss is confined to the ventrolateral substantia nigra leaving other midbrain areas exempts, though it becomes more widespread by the end-stage of the disease, corroborating that neurodegeneration and 
A
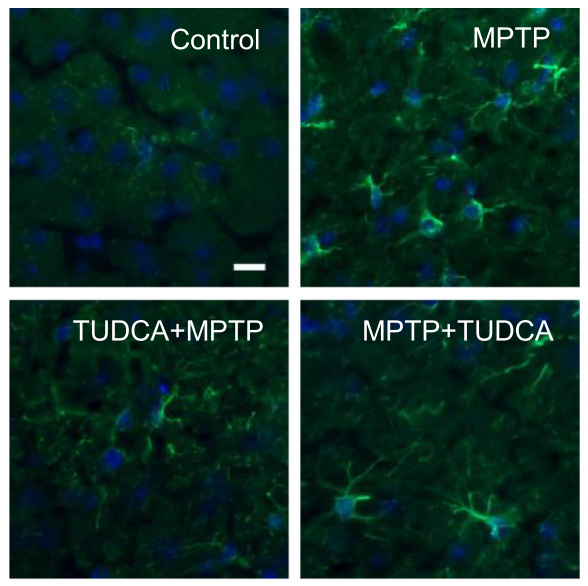

\section{B}
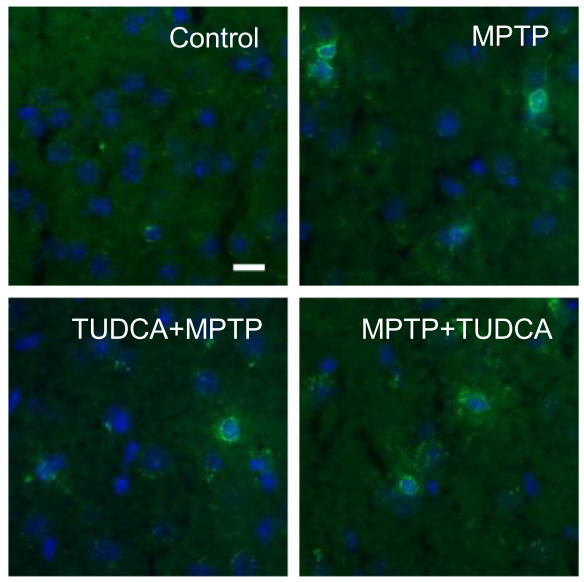

C

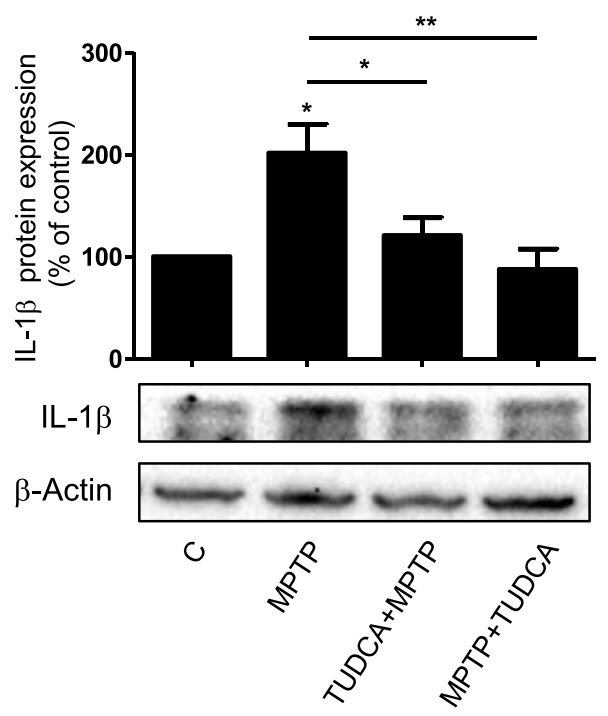



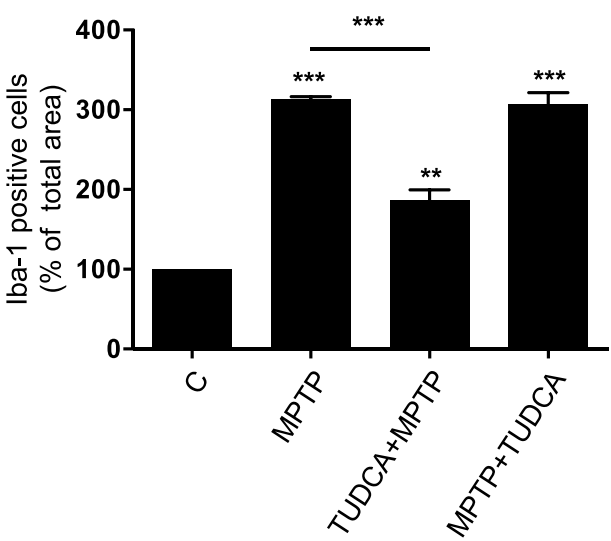

D

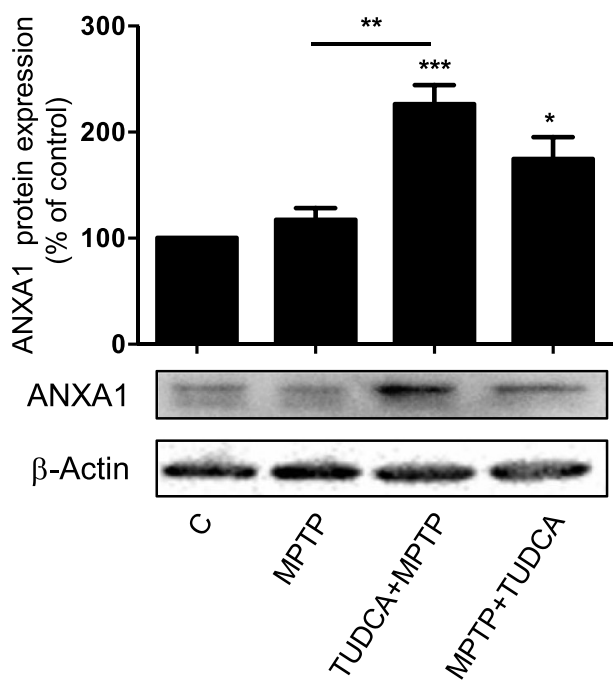

Fig. 4. TUDCA modulates glial activation and ANXA1 expression in the cortex of MPTPtreated mice. Fluorescence microscopy images showing GFAP (A) and Iba-1 (B) staining in the cortex of mice treated with vehicle (control), MPTP, TUDCA prior to MPTP (TUDCA + MPTP) or TUDCA after MPTP (MPTP + TUDCA), euthanized 52 days postMPTP administration. GFAP and Iba-1 are stained in green and nuclei are counterstained with the Hoechst dye 33258. Scale bar $=10 \mu \mathrm{m}$. The area occupied by GFAP or Iba- 1 positive cells was measured using the ImageJ software, and is expressed as percentage of control. Representative immunoblot of IL-1 $\beta$ (C) or ANXA1 (D) in cortex samples from mice treated with vehicle (control), MPTP, TUDCA prior to MPTP (TUDCA + MPTP) or TUDCA after MPTP (MPTP + TUDCA), euthanized 52 days post-MPTP administration. $\beta$-Actin was used as loading control. Relative band intensity was quantified using the Image $\mathrm{Lab}^{\mathrm{TM}}$ software and expressed as a percentage of control. Data shown are mean values \pm SEM of at least three independent experiments. $* p<0.05, * * p<0.01$ and $* * * p<0.001$ using one-way ANOVA with Bonferroni's post hoc test. (For interpretation of the references to colour in this figure legend, the reader is referred to the web version of this article.)
Lewy bodies formation are not only restricted to dopaminergic neurons but also affect noradrenergic, serotonergic and cholinergic systems, as well as the cerebral cortex, olfactory bulb, and autonomic nervous system in PD (Dauer and Przedborski, 2003; Poewe et al., 2017).
Indeed, in the MPTP-induced parkinsonism there is a major loss of serotonin and norepinephrine innervations of the cortex, which may account for the depression symptoms occurring in PD (Nayyar et al., 2009). 
A

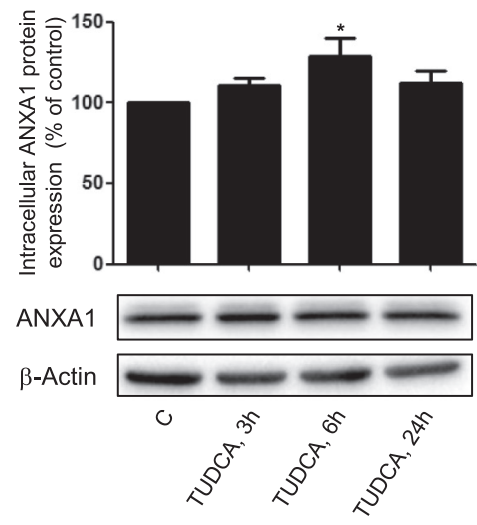

D

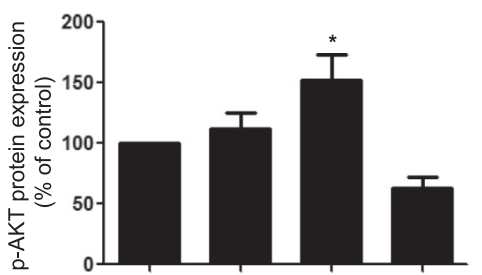

$\mathrm{p}-\mathrm{AKT} \longrightarrow \mathrm{C}$

$\beta$-Actin

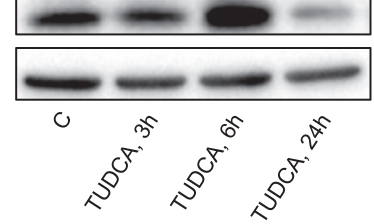

B

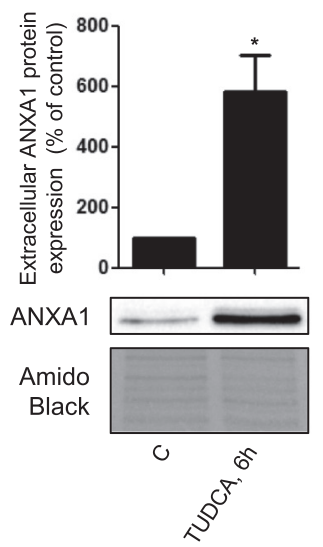

E

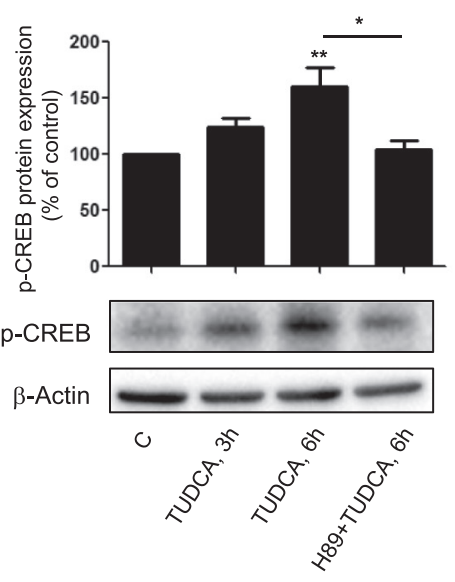

C

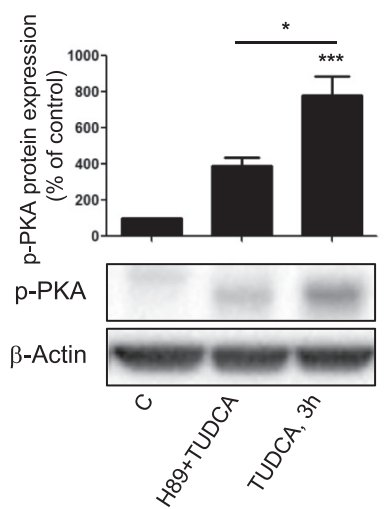

$\mathbf{F}$

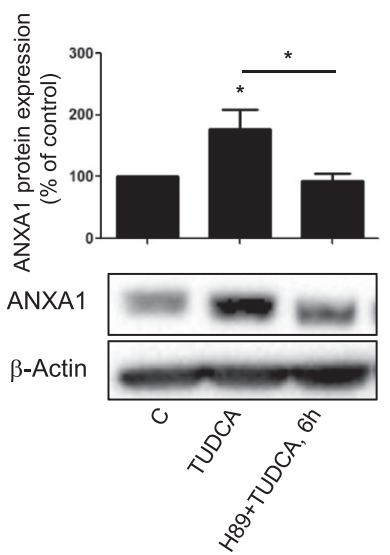

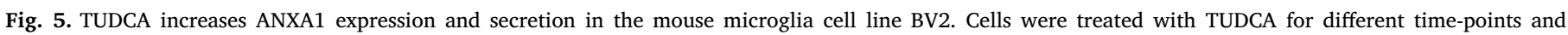

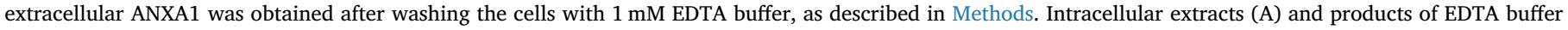

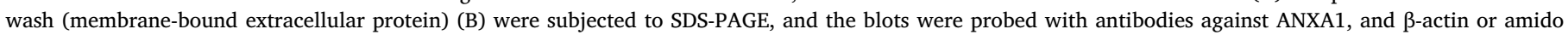

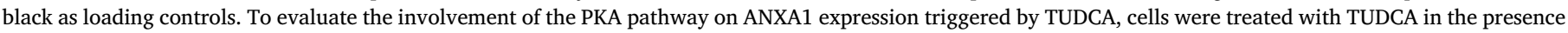

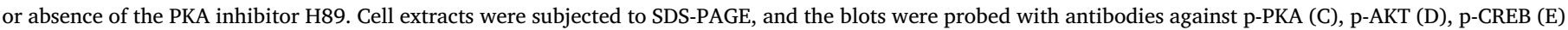

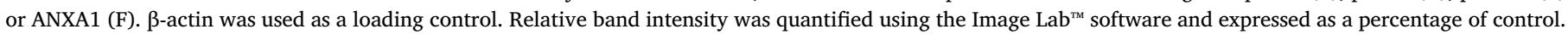

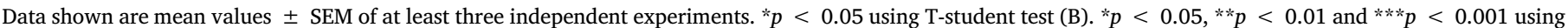
one-way ANOVA with Dunnett's (A, D) or Bonferroni's (C, E and F) post hoc tests.

Increased expression of oxidative stress markers as well as impairment of complex I function were found in cerebral cortex of PD patients (Navarro and Boveris, 2009; Harish et al., 2013; Garcia-Esparcia et al., 2018). Accordingly, adaptive responses including increases in antioxidant enzymes have also been reported in the cortex after MPTP treatment (Cassarino et al., 1997), and cortical dopaminergic denervation has been recently reported in the 6-hydroxydopamine (6OHDA) PD model (Becker et al., 2018).

Here we investigated whether the deleterious effects of MPTP in the mouse brain are also observed in the cerebral cortex. In parallel, we investigated the protective effect of TUDCA in the cortex of mice exposed to MPTP, and in microglia cells.

As an outcome of the neurotoxic/neuroprotective effects of MPTP/ TUDCA in the cerebral cortex, we investigated ATP levels and AMPK activation, as well as endogenous cellular defense mechanisms against oxidative stress. Interestingly, the results presented here recapitulate our previous observations in mice midbrain and striatum (Rosa et al., 2018; Rosa et al., 2017; Moreira et al., 2017), showing that cerebral cortex is also affected in this PD model. In fact, ATP levels were significantly decreased in MPTP treated mice. Moreover, in the short-term treatment, exposure to MPTP and/or treatment with TUDCA increased protein levels of HO-1, GPx1 and parkin. The fact that MPTP by itself up-regulated the expression of these enzymes may indicate a possible compensatory mechanism in response to MPTP-induced oxidative stress, which has already been reported by us in midbrain and striatum (Carvalho et al., 2016; Moreira et al., 2017; Rosa et al., 2017) and by others in the cortex (Cassarino et al., 1997). However, at longer time points increased expression of GPx, HO-1 and parkin were only sustained when animals were pre-treated with TUDCA, since these endogenous adaptive responses triggered after short-term treatment are probably not sufficient nor adequate to overcome the neurodegenerative pathways activated by MPTP. Accordingly, we found that pretreatment with TUDCA also prevented the decrease in ATP levels and promoted AMPK activation. Therefore, pre-treatment with TUDCA not only exacerbates the cytoprotective adaptive responses, but also extends their expression/activation throughout time. These mechanisms may "buffer" the cytotoxic effects of MPTP taking advantage of endogenous pathways, probably responsible for the neuroprotection afforded by this bile acid in the presence of the neurotoxin. These results also highlight the potential of TUDCA as a neuroprotective agent in different cerebral regions, confirming its putative beneficial effect in PD.

To further explore the role of TUDCA in the cerebral cortex, we also investigated its anti-inflammatory effect. As expected, we show an 
increased expression of astro- and microgliosis markers in mice cortex, namely GFAP and Iba-1, respectively, in the presence of MPTP, which is consistent with previous results in the nigro-striatal axis (Rosa et al., 2018; Khan et al., 2013; Lofrumento et al., 2011). Notably, TUDCA treatment decreased the number of GFAP- positive cells, particularly when it was administrated before MPTP. Pre-treatment with TUDCA was also effective in preventing microglia activation, as demonstrated by decreased number of Iba-1 positive cells. Additionally, we show that MPTP increased the expression of IL-1 $\beta$ in mice cortex, which was prevented by pre- or post-treatment with TUDCA. Importantly, our results also show increased ANXA1 expression, when animals were treated with TUDCA either before or after MPTP. Together these results support the anti-inflammatory effect of TUDCA previously described by our group in midbrain and striatum from MPTP-treated mice (Rosa et al., 2018), and by Yanguas-Casas and colleagues in a model of acute neuroinflammation (Yanguas-Casas et al., 2014, 2017).

ANXA1 is an anti-inflammatory protein that in the brain is mostly expressed in microglia cells (de Coupade et al., 2000; McArthur et al., 2010; Solito et al., 2001). Moreover, extracellular ANXA1 has been reported to play a role in anti-inflammatory processes, making the secretion of this protein a possible indicator of a pro-resolving response mediated by ANXA1 (Buckingham and Flower, 1997; Fan et al., 2004; Flower, 1988).

Here we further explored the mechanisms underlying TUDCA modulation of ANXA1 expression using a microglia cell line. The promotor region of ANXA1 gene contains a CRE that can be induced by increased levels of cAMP (Antonicelli et al., 2001; Castro-Caldas et al., 2003; Lima et al., 2017). Interestingly, TUDCA has been shown to mediate anti-inflammatory effects on microglia upon lipopolysaccharide (LPS) administration through activation of the $\mathrm{G}$ proteincoupled bile acid receptor 1 /Takeda $G$ protein-coupled receptor 5 (GPBAR1/TGR5), with consequent increases of intracellular cAMP levels (Yanguas-Casas et al., 2017). Here, we show that TUDCA significantly increased secreted and intracellular levels of ANXA1 in BV2 cells. The up-regulation of ANXA1 secretion, together with de novo synthesis of this protein reveals one possible mechanism by which TUDCA exerts its anti-inflammatory effects, and may explain the results obtained in mouse brain. In dissecting the mechanisms underlying TUDCA modulation of ANXA1 expression, our results show an up-regulation of phosphorylated PKA and CREB transcription factor in cells exposed to TUDCA. Accordingly, we demonstrate a decrease in ANXA1 expression levels in cells that were pre-incubated with the PKA inhibitor, H89. Besides PKA, AKT has also been implicated in CREB activation (Peltier et al., 2007), and we demonstrate that TUDCA significantly up-regulates p-AKT levels. Activation of AKT by TUDCA has already been described by us and others in several cells and models of neurodegenerative diseases and has been associated with its cytoprotective role (Solá et al., 2003; Castro et al., 2004; Castro-Caldas et al., 2012). To our knowledge AKT activation by TUDCA in microglia has never been demonstrated, and further highlights the anti-inflammatory effects of this bile acid. Since PKA and AKT are possible upstream regulators of CREB activation, our data may unveil a pathway responsible for TUDCA regulation of ANXA1 expression that needs to be further explored in vivo, and from a mechanistic point of view.

Other works indicate a time-dependent induction of responses from the nigro-striatal axis to the cerebral cortex. Interestingly, and surprisingly, we found that some of the neuroprotective adaptive responses elicited by MPTP were detected in the same time-points in the nigrostriatal axis and in the cerebral cortex. This is the case for GPx1, HO-1 and parkin. On the other hand, ANXA1 expression was not altered in short-term treatment in the cerebral cortex (data not shown). Moreover, in the cortex GFAP and Iba-1 expression were only increased after longterm treatment with MPTP. Together these findings indicate that different end points have different activation courses in the cerebral cortex and nigro-striatal axis. They also show that the inflammatory reaction is detected at later time-points in the cerebral cortex.
Therefore, our understanding of the disease pathogenic mechanisms should involve a broader analysis of the brain. This study may be a contribution to this emerging approach, adding some new insights to the still scarce data available. In parallel we demonstrate a potential role for ANXA1 upregulation as an anti-inflammatory strategy induced by TUDCA, even though additional work needs to be done to understand the molecular mechanisms involved in this process.

Supplementary data to this article can be found online at https:// doi.org/10.1016/j.mcn.2019.01.003.

\section{Funding}

This work was supported by National funds, through the Foundation for Science and Technology (Portugal) (FCT), under the scope of the projects PTDC/NEU-NMC/0248/2012, UID/DTP/04138/2013 and POCI-01-0145-FEDER-007038, and post-doctoral grants SFRH/ BPD72891/2010 (to A.I.R.), SFRH/BPD/95855/2013 (to M.J.N.), SFRH/BPD/98023/2013 (to A.N.C.), SFRH/BD/102771/2014 (to P.D.) and UMINHO/BI/248/2016 (to S.D.S.). This work has also been developed under the scope of the project NORTE-01-0145-FEDER000013, supported by the Northern Portugal Regional Operational Programme (NORTE 2020), under the Portugal 2020 Partnership Agreement, through the European Regional Development Fund (FEDER), and by FEDER funds, through the Competitiveness Factors Operational Programme (COMPETE).

\section{Conflict of interest}

The authors declare that they have no conflict of interest.

\section{References}

Ackerman, H.D., Gerhard, G.S., 2016. Bile acids in neurodegenerative disorders. Front. Aging Neurosci. 8, 263

Antonicelli, F., De Coupade, C., Russo-Marie, F., Le Garrec, Y., 2001. CREB is involved in mouse annexin A1 regulation by cAMP and glucocorticoids. Eur. J. Biochem. 268 (1), 62-69.

Becker, B., Demirbas, M., Johann, S., Zendedel, A., Beyer, C., Clusmann, H., Haas, S.J., Wree, A., Tan, S.K.H., Kipp, M., 2018. Effect of intrastriatal 6-OHDA lesions on extrastriatal brain structures in the mouse. Mol. Neurobiol. 55 (5), 4240-4252.

Blesa, J., Trigo-Damas, I., Quiroga-Varela, A., Jackson-Lewis, V.R., 2015. Oxidative stress and Parkinson's disease. Front. Neuroanat. 9, 91.

Blum-Degen, D., Müller, T., Kuhn, W., Gerlach, M., Przuntek, H., Riederer, P., 1995 Interleukin-1 beta and interleukin-6 are elevated in the cerebrospinal fluid of Alzheimer's and de novo Parkinson's disease patients. Neurosci. Lett. (1-2), 17-20.

Buckingham, J.C., Flower, R.J., 1997. Lipocortin 1: a second messenger of glucocorticoid action in the hypothalamo-pituitary-adrenocortical axis. Mol. Med. Today 3 (7), 296-302.

Carvalho, A.N., Marques, C., Guedes, R.C., Castro-Caldas, M., Rodrigues, E., van Horssen, J., Gama, M.J., 2016. S-Glutathionylation of Keap1: a new role for glutathione Stransferase pi in neuronal protection. FEBS Lett. 590 (10), 1455-1466.

Cassarino, D.S., Fall, C.P., Swerdlow, R.H., Smith, T.S., Halvorsen, E.M., Miller, S.W. Parks, J.P., Parker Jr., W.D., Bennett Jr., J.P., 1997. Elevated reactive oxygen species and antioxidant enzyme activities in animal and cellular models of Parkinson's disease. Biochim. Biophys. Acta 1362 (1), 77-86.

Castro, R.E., Solá, S., Ramalho, R.M., Steer, C.J., Rodrigues, C.M., 2004. The bile acid tauroursodeoxycholic acid modulates phosphorylation and translocation of bad via phosphatidylinositol 3-kinase in glutamate-induced apoptosis of rat cortical neurons. J. Pharmacol. Exp. Ther. 311 (2), 845-852.

Castro-Caldas, M., Mendes, A.F., Duarte, C.B., Lopes, M.C., 2003. Dexamethasone-induced and estradiol-induced CREB activation and annexin 1 expression in CCRF-CEM lymphoblastic cells: evidence for the involvement of cAMP and p38 MAPK. Mediat. Inflamm. 12 (6), 329-337.

Castro-Caldas, M., Neves, Carvalho A., Peixeiro, I., Rodrigues, E., Lechner, M.C., Gama, M.J., 2009. GSTpi expression in MPTP induced dopaminergic neurodegeneration of C57BL/6 mouse midbrain and striatum. J. Mol. Neurosci. 38 (2), 114-127.

Castro-Caldas, M., Carvalho, A.N., Rodrigues, E., Henderson, C.J., Wolf, C.R., Rodrigues, C.M., Gama, M.J., 2012. Tauroursodeoxycholic acid prevents MPTP-induced dopaminergic cell death in a mouse model of Parkinson's disease. Mol. Neurobiol. 46 (2), 475-486.

Dauer, W., Przedborski, S., 2003. Parkinson's disease: mechanisms and models. Neuron 39 (6), 889-909.

de Coupade, C., Gillet, R., Bennoun, M., Briand, P., Russo-Marie, F., Solito, E., 2000. Annexin 1 expression and phosphorylation are upregulated during liver regeneration and transformation in antithrombin III SV40 T large antigen transgenic mice. Hepatology 31 (2), 371-380. 
Fabre, E., Monserrat, J., Herrero, A., Barja, G., Leret, M.L., 1999. Effect of MPTP on brain mitochondrial $\mathrm{H} 2 \mathrm{O} 2$ and ATP production and on dopamine and DOPAC in the striatum. J. Physiol. Biochem. 55 (4), 325-331.

Fahn, S., 2003. Description of Parkinson's disease as a clinical syndrome. Ann. N. Y. Acad. Sci. $991,1-14$.

Fan, X., Krahling, S., Smith, D., Williamson, P., Schlegel, R.A., 2004. Macrophage surface expression of annexins I and II in the phagocytosis of apoptotic lymphocytes. Mol. Biol. Cell 15 (6), 2863-2872.

Flower, R.J., 1988. Eleventh Gaddum memorial lecture. Lipocortin and the mechanism of action of the glucocorticoids. Br. J. Pharmacol. 94 (4), 987-1015.

Garcia-Esparcia, P., Koneti, A., Rodríguez-Oroz, M.C., Gago, B., Del Rio, J.A., Ferrer, I., 2018. Mitochondrial activity in the frontal cortex area 8 and angular gyrus in Parkinson's disease and Parkinson's disease with dementia. Brain Pathol. 28 (1), 43-57.

Goedert, M., Spillantini, M.G., Del Tredici, K., Braak, H., 2013. 100 years of Lewy pathology. Nat. Rev. Neurol. 9 (1), 13-24.

Guillen, J., 2012. FELASA guidelines and recommendations. J. Am. Assoc. Lab. Anim. Sci. 51 (3), 311-321.

Hagiwara, M., Brindle, P., Harootunian, A., Armstrong, R., Rivier, J., Vale, W., Tsien, R., Montminy, M.R., 1993. Coupling of hormonal stimulation and transcription via the cyclic AMP-responsive factor CREB is rate limited by nuclear entry of protein kinase A. Mol. Cell. Biol. 13 (8), 4852-4859.

Harish, G., Mahadevan, A., Srinivas Bharath, M.M., Shankar, S.K., 2013. Alteration in glutathione content and associated enzyme activities in the synaptic terminals but not in the non-synaptic mitochondria from the frontal cortex of Parkinson's disease brains. Neurochem. Res. 38, 186-200.

Hirsch, E.C., Hunot, S., 2009. Neuroinflammation in Parkinson's disease: a target for neuroprotection? Lancet Neurol. 8 (4), 382-397.

Hurley, S.D., O'Banion, M.K., Song, D.D., Arana, F.S., Olschowka, J.A., Haber, S.N., 2003. Microglial response is poorly correlated with neurodegeneration following chronic, low-dose MPTP administration in monkeys. Exp. Neurol. 184 (2), 659-668.

Hwang, O., 2013. Role of oxidative stress in Parkinson's disease. Exp. Neurobiol. 22 (1), 11-17.

Jackson-Lewis, V., Przedborski, S., 2007. Protocol for the MPTP mouse model of Parkinson's disease. Nat. Protoc. 2 (1), 141-151.

Jackson-Lewis, V., Jakowec, M., Burke, R.E., Przedborski, S., 1995. Time course and morphology of dopaminergic neuronal death caused by the neurotoxin 1-methyl-4 phenyl-1,2,3,6-tetrahydropyridine. Neurodegeneration 4 (3), 257-269.

Keene, C.D., Rodrigues, C.M., Eich, T., Linehan-Stieers, C., Abt, A., Kren, B.T., Steer, C.J., Low, W.C., 2001. A bile acid protects against motor and cognitive deficits and reduces striatal degeneration in the 3-nitropropionic acid model of Huntington's disease. Exp. Neurol. 171 (2), 351-360.

Khan, M.M., Kempuraj, D., Thangavel, R., Zaheer, A., 2013. Protection of MPTP-induced neuroinflammation and neurodegeneration by Pycnogenol. Neurochem. Int. 62 (4), 379-388.

Klein, C., Westenberger, A., 2012. Genetics of Parkinson's disease. Cold Spring Harb. Perspect. Med. 2 (1), a008888.

Liberatore, G.T., Jackson-Lewis, V., Vukosavic, S., Mandir, A.S., Vila, M., McAuliffe, W.G., Dawson, V.L., Dawson, T.M., Przedborski, S., 1999. Inducible nitric oxide synthase stimulates dopaminergic neurodegeneration in the MPTP model of Parkinson disease. Nat. Med. 5 (12), 1403-1409.

Liddelow, S.A., Guttenplan, K.A., Clarke, L.E., Bennett, F.C., Bohlen, C.J., Schirmer, L., Bennett, M.L., Munch, A.E., et al., 2017. Neurotoxic reactive astrocytes are induced by activated microglia. Nature 541 (7638), 481-487.

Lima, K.M., Vago, J.P., Caux, T.R., Negreiros-Lima, G.L., Sugimoto, M.A., Tavares, L.P., Arribada, R.G., Carmo, A.A.F., Galvao, I., Costa, B.R.C., Soriani, F.M., Pinho, V., Solito, E., Perretti, M., Teixeira, M.M., Sousa, L.P., 2017. The resolution of acute inflammation induced by cyclic AMP is dependent on annexin A1. J. Biol. Chem. 292 (33), 13758-13773.

Lofrumento, D.D., Saponaro, C., Cianciulli, A., De Nuccio, F., Mitolo, V., Nicolardi, G., Panaro, M.A., 2011. MPTP-induced neuroinflammation increases the expression of pro-inflammatory cytokines and their receptors in mouse brain. Neuroimmunomodulation 18 (2), 79-88.

Martin, I., Dawson, V.L., Dawson, T.M., 2011. Recent advances in the genetics of Parkinson's disease. Annu. Rev. Genomics Hum. Genet. 12, 301-325.

McArthur, S., Cristante, E., Paterno, M., Christian, H., Roncaroli, F., Gillies, G.E., Solito, E., 2010. Annexin A1: a central player in the anti-inflammatory and neuroprotective role of microglia. J. Immunol. 185 (10), 6317-6328.

McGeer, P.L., Itagaki, S., Boyes, B.E., McGeer, E.G., 1988. Reactive microglia are positive for HLA-DR in the substantia nigra of Parkinson's and Alzheimer's disease brains. Neurology 38 (8), 1285-1291.
Miklossy, J., Doudet, D.D., Schwab, C., Yu, S., McGeer, E.G., McGeer, P.L., 2006. Role of ICAM-1 in persisting inflammation in Parkinson disease and MPTP monkeys. Exp. Neurol. 197 (2), 275-283.

Mogi, M., Harada, M., Kondo, T., Riederer, P., Inagaki, H., Minami, M., Nagatsu, T., 1994. Interleukin-1 beta, interleukin-6, epidermal growth factor and transforming growth factor-alpha are elevated in the brain from parkinsonian patients. Neurosci. Lett. 180 (2), 147-150.

Moon, H.E., Paek, S.H., 2015. Mitochondrial dysfunction in Parkinson's disease. Exp. Neurobiol. 24 (2), 103-116.

Moreira, S., Fonseca, I., Nunes, M.J., Rosa, A., Lemos, L., Rodrigues, E., Carvalho, A.N., Outeiro, T.F., Rodrigues, C.M.P., Gama, M.J., Castro-Caldas, M., 2017. Nrf2 activation by tauroursodeoxycholic acid in experimental models of Parkinson's disease. Exp. Neurol. 295, 77-87.

Muller, T., Blum-Degen, D., Przuntek, H., Kuhn, W., 1998. Interleukin-6 levels in cerebrospinal fluid inversely correlate to severity of Parkinson's disease. Acta Neurol. Scand. 98 (2), 142-144.

Navarro, A., Boveris, A., 2009. Brain mitochondrial dysfunction and oxidative damage in Parkinson's disease. J. Bioenerg. Biomembr. 41, 517-521.

Nayyar, T., Bubser, M., Ferguson, M.C., Neely, M.D., Shawn Goodwin, J., Montine, T.J., Deutch, A.Y., Ansah, T.A., 2009. Cortical serotonin and norepinephrine denervation in parkinsonism: preferential loss of the beaded serotonin innervation. Eur. J. Neurosci. 30 (2), 207-216.

Nicklas, W.J., Vyas, I., Heikkila, R.E., 1985. Inhibition of NADH-linked oxidation in brain mitochondria by 1-methyl-4-phenyl-pyridine, a metabolite of the neurotoxin, 1-methyl-4-phenyl-1,2,5,6-tetrahydropyridine. Life Sci. 36 (26), 2503-2508.

Nicklas, W., Baneux, P., Boot, R., Decelle, T., Deeny, A.A., Fumanelli, M., Illgen-Wilcke, B., Felasa, 2002. Recommendations for the health monitoring of rodent and rabbit colonies in breeding and experimental units. Lab. Anim. 36 (1), 20-42.

Niranjan, R., 2014. The role of inflammatory and oxidative stress mechanisms in the pathogenesis of Parkinson's disease: focus on astrocytes. Mol. Neurobiol. 49 (1), 28-38.

Peltier, J., O'Neill, A., Schaffer, D.V., 2007. PI3K/Akt and CREB regulate adult neural hippocampal progenitor proliferation and differentiation. Dev. Neurobiol. 67 (10), 1348-1361.

Poewe, W., Seppi, K., Tanner, C.M., Halliday, G.M., Brundin, P., Volkmann, J., Schrag, A.E., Lang, A.E., 2017. Parkinson disease. Nat. Rev. Dis. Primers. 3, 17013.

Pringsheim, T., Jette, N., Frolkis, A., Steeves, T.D., 2014. The prevalence of Parkinson's disease: a systematic review and meta-analysis. Mov. Disord. 29 (13), 1583-1590.

Przedborski, S., 2005. Pathogenesis of nigral cell death in Parkinson's disease. Parkinsonism Relat. Disord. 11 (Suppl. 1), S3-S7.

Rodrigues, C.M., Spellman, S.R., Sola, S., Grande, A.W., Linehan-Stieers, C., Low, W.C., Steer, C.J., 2002. Neuroprotection by a bile acid in an acute stroke model in the rat. J. Cereb. Blood Flow Metab. 22 (4), 463-471.

Rosa, A.I., Fonseca, I., Nunes, M.J., Moreira, S., Rodrigues, E., Carvalho, A.N., Rodrigues, C.M.P., Gama, M.J., Castro-Caldas, M., 2017. Novel insights into the antioxidant role of tauroursodeoxycholic acid in experimental models of Parkinson's disease. Biochim. Biophys. Acta 1863 (9), 2171-2181.

Rosa, A.I., Duarte-Silva, S., Silva-Fernandes, A., Nunes, M.J., Carvalho, A.N., Rodrigues, E., Gama, M.J., Rodrigues, C.M.P., Maciel, P., Castro-Caldas, M., 2018. Tauroursodeoxycholic acid improves motor symptoms in a mouse model of Parkinson's disease. Mol. Neurobiol. 55 (12), 9139-9155.

Saporito, M.S., Thomas, B.A., Scott, R.W., 2000. MPTP activates c-Jun NH(2)-terminal kinase (JNK) and its upstream regulatory kinase MKK4 in nigrostriatal neurons in vivo. J. Neurochem. 75 (3), 1200-1208.

Solá, S., Castro, R.E., Laires, P.A., Steer, C.J., Rodrigues, C.M., 2003. Tauroursodeoxycholic acid prevents amyloid-beta peptide-induced neuronal death via a phosphatidylinositol 3-kinase-dependent signaling pathway. Mol. Med. 9 (9-12), 226-234.

Solito, E., de Coupade, C., Canaider, S., Goulding, N.J., Perretti, M., 2001. Transfection of annexin 1 in monocytic cells produces a high degree of spontaneous and stimulated apoptosis associated with caspase-3 activation. Br. J. Pharmacol. 133 (2), 217-228.

Solito, E., McArthur, S., Christian, H., Gavins, F., Buckingham, J.C., Gillies, G.E, 2008. Annexin A1 in the brain-undiscovered roles? Trends Pharmacol. Sci. 29 (3), $135-142$.

Thomas, B., Flint, Beal M., 2007. Parkinson's disease. Hum. Mol. Genet. 16 (R2), 183-194.

Yanguas-Casas, N., Barreda-Manso, M.A., Nieto-Sampedro, M., Romero-Ramirez, L., 2014. Tauroursodeoxycholic acid reduces glial cell activation in an animal model of acute neuroinflammation. J. Neuroinflammation 11, 50.

Yanguas-Casas, N., Barreda-Manso, M.A., Nieto-Sampedro, M., Romero-Ramirez, L., 2017. TUDCA: an agonist of the bile acid receptor GPBAR1/TGR5 with anti-inflammatory effects in microglial cells. J. Cell. Physiol. 232 (8), 2231-2245. 